\title{
Hyperthyroidism caused by a pituitary adenoma
}

\author{
Thomas Arnason MD, David B. Clarke MDCM PhD, Syed Ali Imran MBBS
}

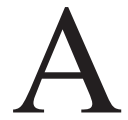

49-year-old woman was referred for hyperthyroidism that was difficult to control despite methimazole therapy. Her symptoms included headaches, palpitations, left-eye pain and frequent loose bowel movements. At referral, her serum thyroidstimulating hormone (TSH) level was 10.45 (normal 0.35-5.5) $\mathrm{mIU} / \mathrm{L}$ and her free thyroxine $\left(\mathrm{FT}_{4}\right)$ level was 17.8 (normal 11.5-22.7) $\mathrm{pmol} / \mathrm{L}$. A two-week methimazole withdrawal was attempted, resulting in worsening symptoms. Repeat blood work showed a high-normal TSH level (5.21 mIU/L) and an elevated $\mathrm{FT}_{4}$ level (27.2 pmol/L). Magnetic resonance imaging (MRI) of the sella turcica showed a $1.2-\mathrm{cm}$ pituitary mass (Figure 1). She underwent endoscopic transnasal transsphenoidal resection. ${ }^{1}$ Pathologic examination of the resected mass (Appendix 1, available at www.cmaj.ca/lookup/ suppl/doi:10.1503/cmaj.101244/-/DC1) was diagnostic of a TSH-producing pituitary adenoma. Her symptoms resolved following surgery. Three weeks after surgery, blood tests showed a TSH level of $1.19 \mathrm{mIU} / \mathrm{L}$ and an $\mathrm{FT}_{4}$ level of $9.7 \mathrm{pmol} / \mathrm{L}$. Levothyroxine replacement was started, and three months later the patient was biochemically and clinically euthyroid. Repeat MRI showed complete tumour excision.

The differential diagnosis for inappropriate secretion of TSH with high $\mathrm{FT}_{4}$ includes interference from heterophilic antibodies and resistance to thyroid hormone. Interference from heterophilic antibodies can be ruled out using various laboratory strategies. Resistance to thyroid hormone can be ruled out using biochemical tests including the $\alpha$-subunit:TSH molar ratio, thyrotropin-releasing hormone administration test and sex hormone binding globulin test. ${ }^{2}$ Resistance to TSH, in which the TSH level is elevated but $\mathrm{FT}_{4}$ levels are decreased, should not be confused with resistance to thyroid hormone. Although MRI can provide anatomic evidence of a pituitary adenoma, pituitary enlargement may also occur in hypothyroid patients because of thyrotroph hyperplasia. The gold standard method for diagnosis of TSH-producing pitu-

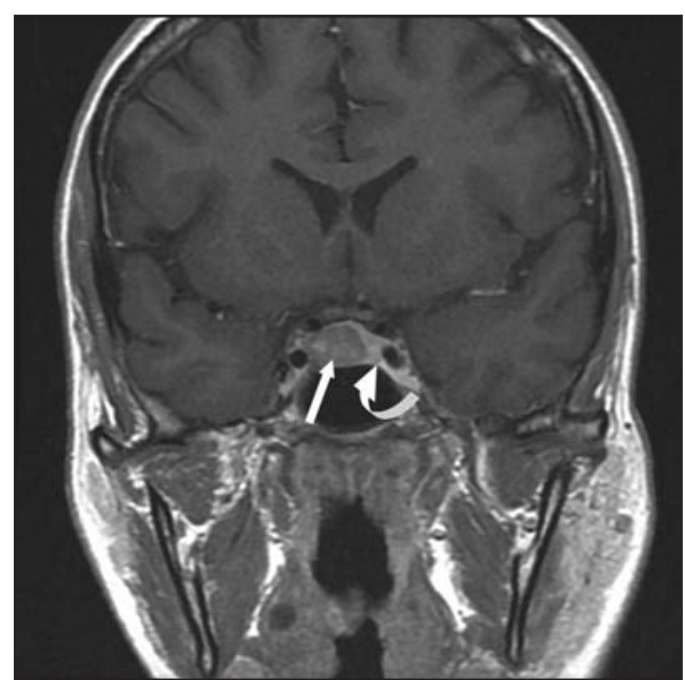

Figure 1: Coronal $T_{1}$-weighted magnetic resonance image (with gadolinium enhancement) in a 49-yearold woman, showing a hypointense macroadenoma (straight arrow) filling the centre and right side of the sella turcica. The enhancing normal pituitary tissue (curved arrow) is displaced to the left.

itary adenoma remains pathologic examination of the tumour. ${ }^{2}$

Pituitary adenomas that produce TSH represent only $1 \%$ of functioning pituitary adenomas. ${ }^{3}$ Patients most commonly present with symptoms of hyperthyroidism or diffuse goiter. ${ }^{3}$ Although surgery is definitive, other treatment options in symptomatic patients include radiotherapy and medical management. A review of long-acting somatostatin analogue treatment in eight studies found normalization of thyroid hormone in $95 \%$ of patients and reduction in tumour size in $40 \%{ }^{4}$

\section{References}

1. McGrath BM, Maloney WJ, Wolfsberger S, et al. Carotid artery visualization during anterior skull base surgery: a novel protocol for neuronavigation. Pituitary 2010;13:215-22.

2. Khandwala H, Lee C. Inappropriate secretion of thyroidstimulating hormone. CMAJ 2006; 175:351-3.

3. DeLellis RA, Lloyd RV, Heitz PU, et al., editors. World Health Organization classification of tumours. Pathology \& genetics: tumours of endocrine organs. Lyon (FR): IARC Press; 2004. p. 9-39.

4. Beck-Peccoz P, Persani L, Mannavola D, et al. Pituitary tumours: TSH-secreting adenomas. Best Pract Res Clin Endocrinol Metab 2009;23:597-606.
Competing interests: None declared.

This article has been peer reviewed.

Affiliations: From the Department of Laboratory Medicine, Division of Anatomic Pathology (Arnason), the Department of Surgery, Division of Neurosurgery (Clarke) and the Department of Medicine, Division of Endocrinology (Imran), Queen Elizabeth II Health Sciences Centre, Dalhousie University, Halifax, NS

Correspondence to: Dr. Thomas Arnason, tarnason@dal.ca

CMAJ 2011. DOI:10.1503 /cmaj.101244 